Goddard Space Flight Center

NATIONAL AERONAUTICS AND SPACE ADMINISTRATION WASHINGTON 

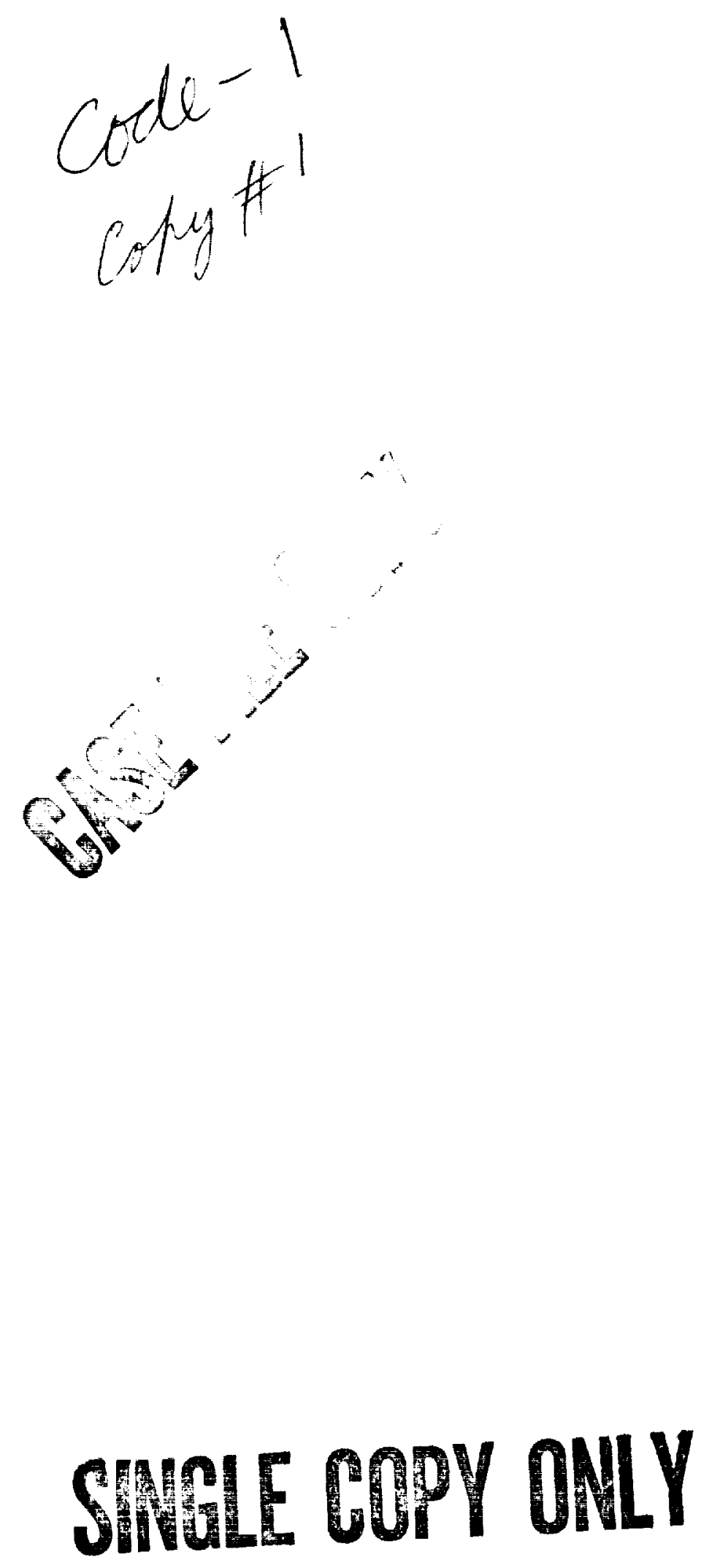


\title{
THE LOW FREQUENCY POWER SPECTRUM OF COSMIC-RAY VARIATIONS DURING IGY
}

\author{
by \\ David Stern \\ Goddard Space Flight Center
}

\section{SUMMARY}

The power spectra of cosmic-ray variations, as recorded by neutron monitors at various locations, is computed and analyzed herein. The frequency range of the variations covered (from 1/200 to $1 / 2$ per day) is found to contain only one significant peak, contributed by the well known 27-day variation. By use of the power spectrum, a quantitative estimate of the latitude dependence of the 27-day variation may be made. From this, the average rigidity dependence of the modulation amplitude is deduced, and is found to be essentially the same as that of Forbush decreases. The average was taken over the period July 1957 to December 1958, during which the peak decreased significantly.

Irregular variations were also investigated, as was the correlation with magnetic activity. It is found that the irregular variations have approximately the same latitude dependence as the 27-day peak, decreasing roughly exponentially with increasing frequency. There was some correlation between magnetic activity and cosmic-ray variations, but it showed no clear recurrence tendencies.

A general review of power spectral analysis, with emphasis on points relevant to this work, is included here, and the conclusions along with the connection between Forbush decreases and the 27-day variation are discussed. 

CONTENTS

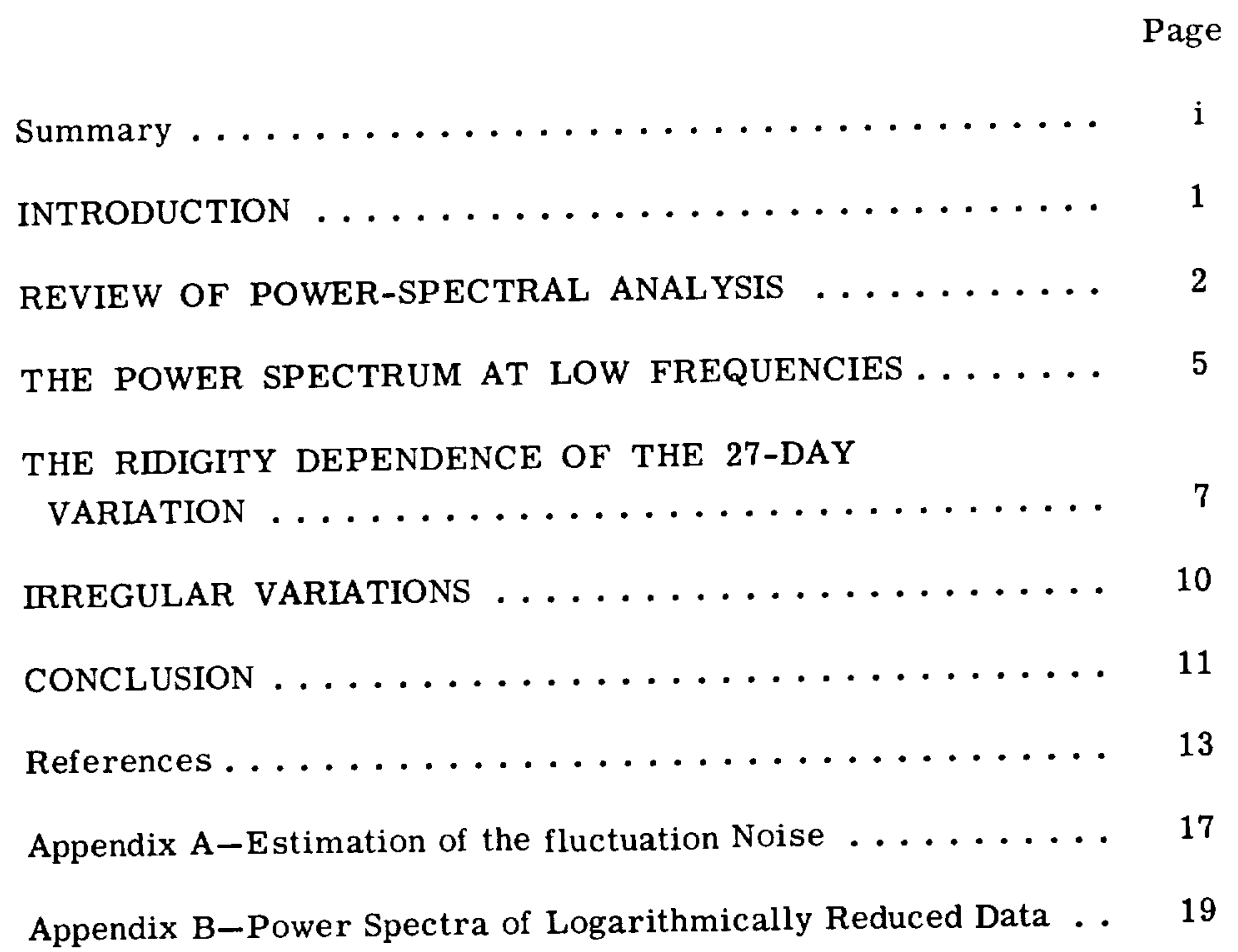





\title{
THE LOW FREQUENCY POWER SPECTRUM OF COSMIC-RAY VARIATIONS DURING IGY*
}

\author{
by \\ David Stern ${ }^{\dagger}$ \\ Goddard Space Flight Center
}

\section{INTRODUCTION}

Examination of the record of a high counting rate cosmic-ray neutron monitor immediately reveals its large variability. At least four definite sources of variation can be traced: the 11-year cycle; the Forbush decreases accompanying sudden commencement (SC) magnetic storms; the 27-day variation; and the daily variation. Of these, three are periodic in character: (1) the 11-year variation, which is connected with the cycle of solar activity and will not be discussed further; (2) the 27day variation, which is believed to be associated with the period of solar rotation; and (3) the daily variation, which reflects anisotropy in the cosmic radiation reaching the earth. Both the 27-day and the daily variation are often observed to undergo large changes in amplitude within a few cycles. In addition, the 27-day variation seems to vary in both phase and frequency (References 1,2 and 3). If the sources of the 27-day periodicity are located upon the sun, such changes are indeed expected since all features of the solar surface have a transient nature. Thus, a source of 27-day variation may in time be superseded by another, at a different latitude (which affects the rotation period), and at a different longitude (which affects the phase). Changes of this kind, unfortunately, complicate the study of the phenomenon.

Apart from these well-defined variations, the counting rate undergoes various irregular fluctuations. Comparison between stations confirms the fact that these are genuine fluctuations, and not of instrumental origin. Some of the questions associated with these fluctuations are:

(1) What is their typical time scale?

(2) What is their energy dependence?

(3) Do there exist in the primary flux any periodic variations other than those listed?

To answer these and similar questions, and to analyze phase-unstable periodic variations quantitatively, the method of power spectrum analysis is very useful. This method will now be briefly reviewed.

* Published in substantially the same form in J. Geopbys. Res. 67:2133-2144, June 1962.

†his work was performed under a National Academy of Sciences postdoctoral scholarship. 


\section{REVIEW OF POWER-SPECTRAL ANALYSIS}

Conventional Fourier analysis is not suitable for the analysis of a time-dependent counting rate $x(t)$, which tends to be periodic with frequency $f_{0}$, but randomly changes its phase, now and again. Indeed, it can be shown that as the length of the given record tends to infinity, the corresponding estimated Fourier transform of $x(t)$ for any frequency approaches zero. This is true even for $f_{0}$; over the long run, $x(t)$ will have equal probability for being in or out of phase. Two main approaches exist for frequency analysis in this case.

One approach is based on the fact that even though the Fourier transform of $x(t)$ approaches zero in the limit, its mean square, under very general assumptions, tends to a finite limit $P(f)$ :

$$
P(f)=\lim _{T \rightarrow \infty} \frac{1}{T}\left|\int_{-T / 2}^{T / 2} x(t) e^{2 \pi f i t} d t\right|^{2}
$$

The function $P(f)$ is called the power spectral density, and will be strongly peaked at $f=f_{0}$. It differs only in minor details from the "Periodogram" introduced by A. Schuster (Reference 4; also see, for instance, Reference 5, Section 16.30). It can be intuitively understood as follows: let $x(t)$ be regarded as a voltage signal, and let it be passed through a filter network sharply tuned to pass only a narrow band $\Delta f$ around the frequency $f$. Let the output signal be fed into a resistance of one ohm. The mean power of the output signal, which is proportional to the mean square of the output voltage, will then be $P(f) \Delta f$ (hence the name power spectrum). Power spectral analysis of a time-varying quantity is, therefore, similar to analysis of an unknown voltage signal for its frequency content by means of frequency filters (Reference 6).

An alternative approach is the investigation of recurrence tendencies. An early method attributed to Chree (Reference 7) consists of selecting, according to a predetermined criterion, times at which $x(t)$ was highest (or lowest), and then superimposing the record upon itself so that all selected points overlap. If there is a tendency for a maximum (or minimum) of $x(t)$ to recur after a period $\tau$, this will generally show up in the sum of the superimposed records. This method has clearly demonstrated, among other things, the 27-day periodicity in cosmic-ray variations (see, for example, References $2,3,8,9,10$, and 11). Unfortunately, it is not suitable for quantitative evaluations.

A more satisfactory measure for recurrence is the autocorrelation or autocovariance function, defined as:

$$
C(\tau)=\lim _{T-\infty} \frac{1}{\bar{T}} \int_{-T / 2}^{T / 2}[x(t)-\bar{x}][x(t-\tau)-\bar{x}] d t
$$

If we first normalize $x(t)$ so that $\bar{x}=0$, then $C(\tau)$ simplifies to

$$
C(\tau)=\overline{x(t) \times(t-\tau)} .
$$


Assuming that the process is symmetric about its mean, $x(t)$ has equal probability to be of either sign. The same holds for $x(t-\tau)$, provided it is totally unrelated to $x(t)$; in this case $c(\tau)$ will, over a long run, approach zero. On the other hand, if $\tau$ represents a recurrence period, whatever the sign of $x(t)$, then $x(t-\tau)$ will have more than even probability of being of the same sign, so that $C(\tau)$ tends to a positive limit.

Wiener and Khintchine (Reference 12, footnote 16) showed that the two measures described here contain equivalent information, and each can be derived from the other by means of a Fourier transformation:

$$
\begin{aligned}
& \mathrm{C}(\tau)=\int_{-\infty}^{\infty} \mathrm{P}(\mathrm{f}) \mathrm{e}^{2 \pi \mathrm{if} \tau} \mathrm{df} \\
& \mathrm{P}(\mathrm{f})=\int_{-\infty}^{\infty} \mathrm{C}(\tau) \mathrm{e}^{-2 \pi \mathrm{i} f \tau} \mathrm{d} \tau .
\end{aligned}
$$

The ordinary method of estimating the power spectrum, though not the only one (see, for instance, Reference 13), therefore involves prior estimation of the autocorrelation function by means of the finite record on hand, from which an estimate of $\mathrm{P}(\mathrm{f})$ is obtained by transformation. Though both functions contain equivalent information, it is useful to consider both for complete understanding of the behavior of $x(t)$. If there is a recurrence tendency with period $\tau_{0}$ lasting more than one cycle, $\mathrm{C}(\tau)$ will have peaks not only at $\tau_{0}$ but also at $2 \tau_{0}, 3 \tau_{0}$, etc.; it will, therefore, indicate the average number of oscillations between phase jumps. On the other hand, $P(f)$ has the advantage of concentrating all the information about the component with frequency $f$ at one point. This is especially important when there is more than one frequency involved, in which case, $C(\tau)$ is often rather irregular.

More details can be found in a number of books and reviews dealing with the subject (References $12,14,15,16,17,18,19$ and 20). Of these, the one by Blackman and Tukey (Reference 18) is most useful in dealing with the practical problems connected with actual estimation of the power spectrum; the present computation, using SHARE program 574 adapted for the 7090 computer, essentially follows their method. Some relevant points, quoted here without details, are the following:

Frequency Resolution: If the data are sampled at intervals $\Delta t, \mathrm{C}(\tau)$ can only be estimated for integral multiples of $\Delta t$. Furthermore, if $\tau_{\max }=m \Delta t$ so that $\mathrm{C}(\tau)$ is estimated for $m+1$ values of $\tau$, the resulting estimates of $P(f)$ will cover $m+1$ points, equally spaced in frequency, from zero to $f_{\max }=1 / 2 \Delta t$. Each estimate of $P(f)$ will represent an average of the power spectrum over a band of the order $\mathrm{f}_{\mathrm{min}}=1 / 2 \mathrm{~m} \Delta t$ around the frequency it represents; the exact width and shape of the band depend on the relative weights given to the estimates of $\mathrm{C}(\tau)$. "Hanning windows" were used in this case. In the present computation, daily averages of the cosmic-ray intensity were used and usually 100 estimates were taken, covering the spectrum for periods between 2 and 200 days.

Aliasing: If the power spectrum does not vanish above $\mathrm{f}_{\max }$, higher frequencies will contribute to the estimated spectrum in a way which cannot be resolved (aliasing). The spectrum of cosmic-ray variations has been found to fall off with increasing frequency rapidly enough as to make this source 
of error negligible. Even if this were not so, the fact that data points represent daily averages, and not momentary samples taken at daily intervals, strongly suppresses contributions from higher frequencies.

The Accuracy of Estimation: The method here described assumes that the process is stationary in time - i.e., insensitive to a shift of the time axis, and this assumption may be only an approximation. Indeed, the power spectral density of cosmic-ray variations changes over the period investigated, and therefore, any estimate of it gives only a time-averaged result (Reference 21). Secondly, the question arises as to how closely the spectral estimates drawn from a limited sample approximate the actual values. Blackman and Tukey show that the ratio between a sample obtained by $m$ estimates from $\mathrm{n}$ data points, and the actual value, approximately follows a $x^{2}$ distribution $w i t h \nu=2 \mathrm{n} / \mathrm{m}$ degrees of freedom. For analyses covering the IGY period (July 1957 to December 1958), $\mathrm{n}=549$, giving $\nu=11$. Finally, it should be remembered that because of the statistical fluctuations in the counting rate, our record is not only limited in length, but also has not been sampled with ideal accuracy. It can be shown, however, (Appendix A) that at least in the present case, this source of error is totally negligible.

Power spectrum analysis has been used in the investigation of diverse processes, such as the free oscillations of the earth, recurrence of magnetic storms, frequency analysis of sea waves, turbulence, and many others. Only in a few cases, however, has it been used to analyze cosmic-ray

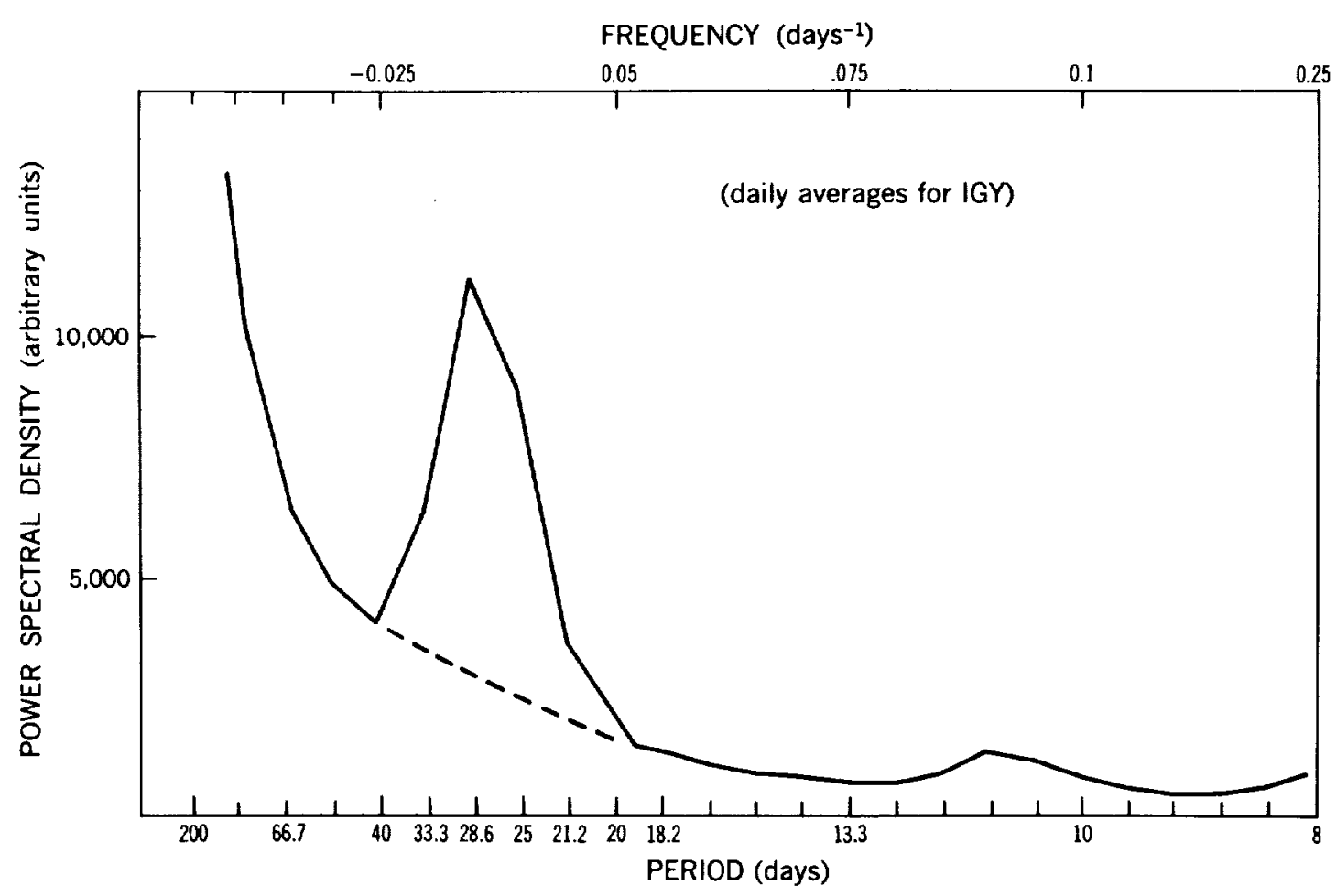

Figure 1-The low frequency power spectrum of cosmic-ray variations during IGY as recorded by the Climax neutron monitor. 
variations. Fonger (Reference 22) used the autocorrelation to demonstrate a 27 -day recurrence, but since his data covered only a three month period, they are not very significant statistically. Panofsky, Lethbridge and Neuberger (Reference 23) obtained power spectra of neutron monitor rates, and cross correlated them with various meteorological data. In the present work, an attempt has been made to deduce detailed properties of low frequency variations of the cosmic-ray intensity (as measured by neutron monitors during IGY), and especially of the 27-day variation.

\section{THE POWER SPECTRUM AT LOW FREQUENCIES}

As Figure 1 shows, the power-spectral density exhibits a marked peak between 27 and 28 days. The location of the peak is not fixed (see Figure 2), and over the period July 1957 - December 1959 its variation does not exhibit any marked trend (cf. Reference 2, Table III). None of the other peaks in the spectrum is believed to be significant; in particular, the absence of a conspicuous second harmonic is noted. Throughout the period analyzed, the amplitude of the 27-day peak rapidly decreases with time, as can be seen in Figure 2 . The results described here are averages computed over the IGY period; during the first half year of the IGY, the amplitudes were about twice this average magnitude.

The autocorrelation has been plotted at daily intervals for 200 days (see Figure 3 ), and it is evident that the recurrence tendency is relatively stable.

The counting rate was also cross-correlated with the magnetic activity $C$ indices, tabulated in the reports of Solar-Geophysical databy the National Bureau of Standards. A sharp negative peak is observed in the cross-correlation function:

$$
C_{c}(\tau)=\overline{X(t) Y(t-\tau)}
$$

where $X(t)$ is the counting rate and $Y(t)$ is the magnetic activity index. It is located near $t=1$ day (see Figure 4), indicating that high geomagnetic activity is likely to be followed within one day (on the average) by low cosmic-ray rates. This agrees with known properties of SC magnetic storms; a 27-day recurrence tendency was

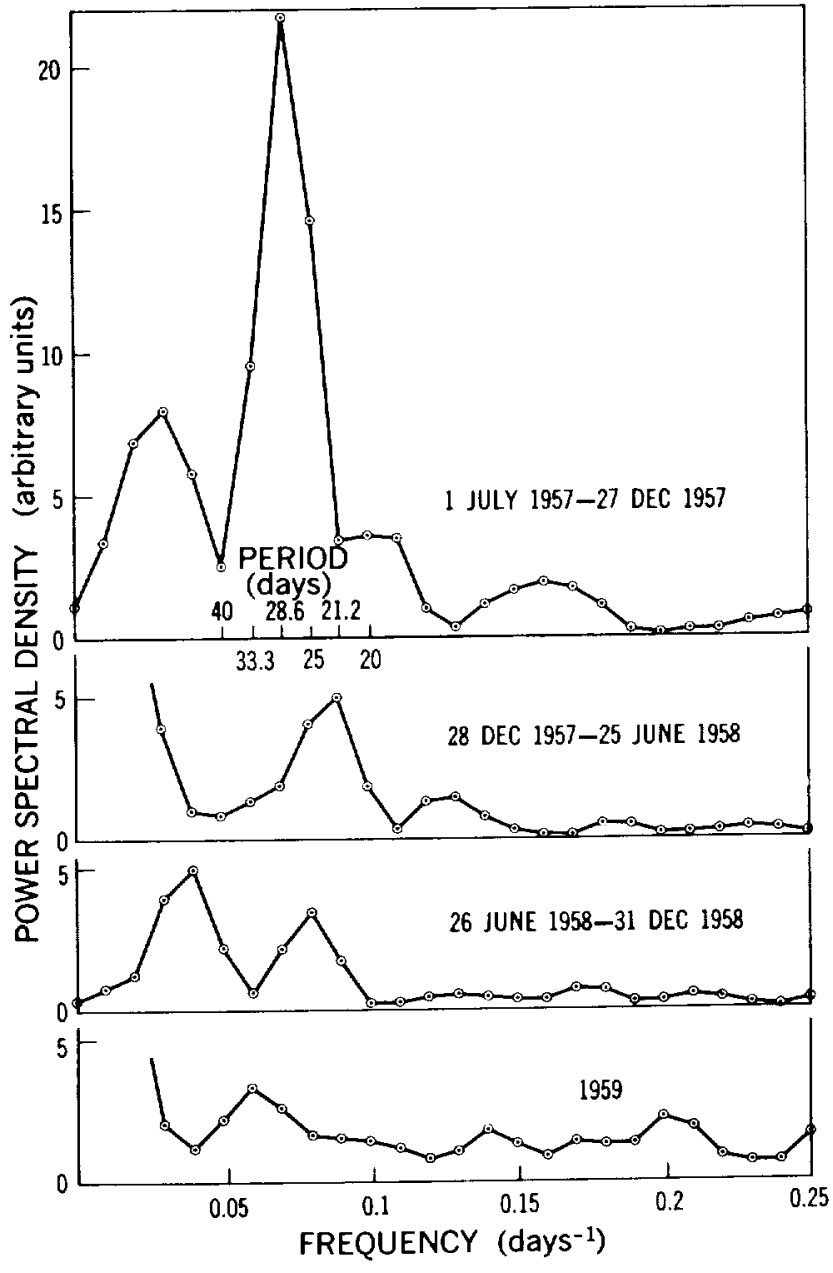

Figure 2-The power spectrum of cosmic-ray variations as recorded by the Zugspitze neutron monitor for different parts of IGY and for 1959 


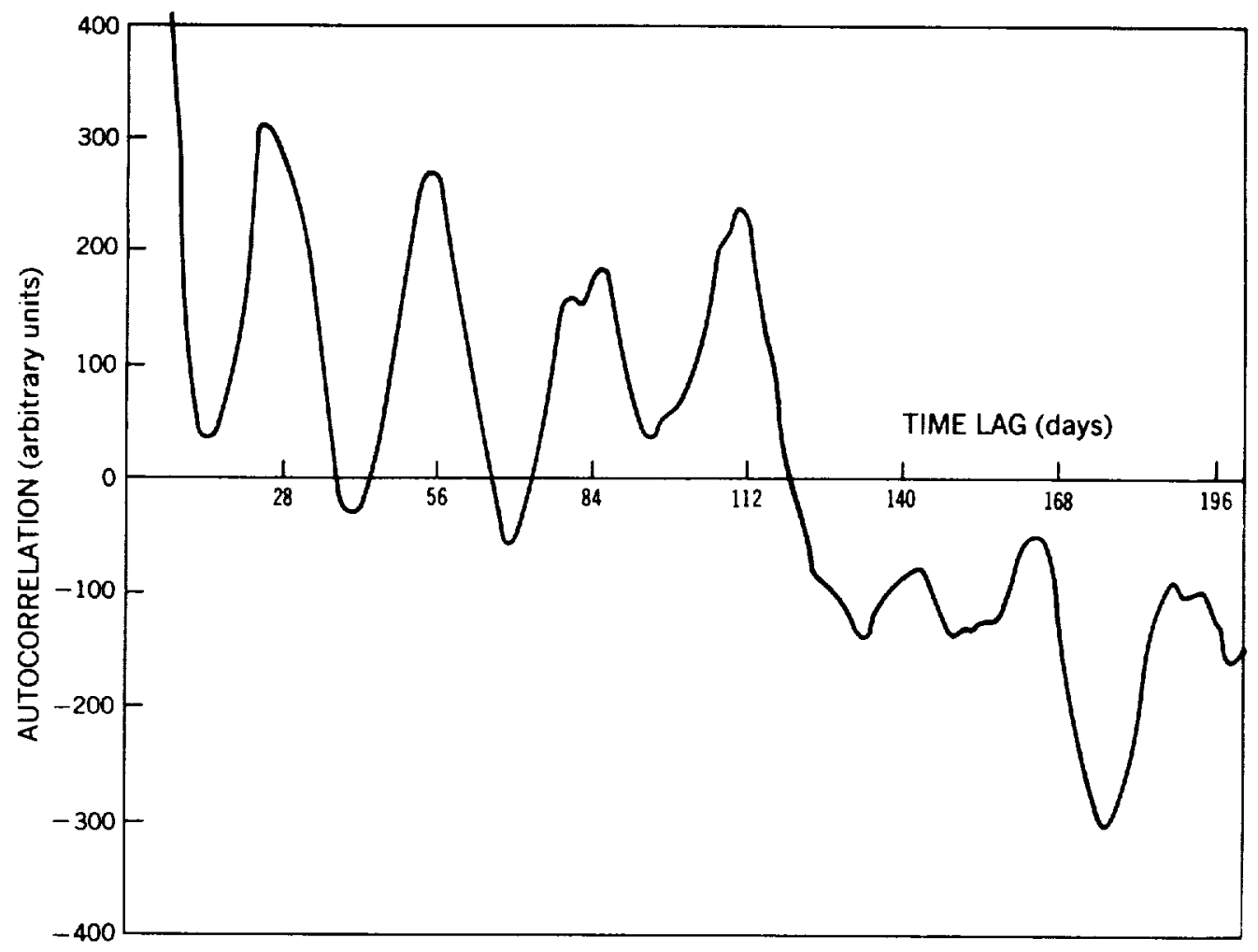

Figure 3-The autocorrelation function of cosmic-ray variations during IGY as recorded by the Climax neutron monitor.

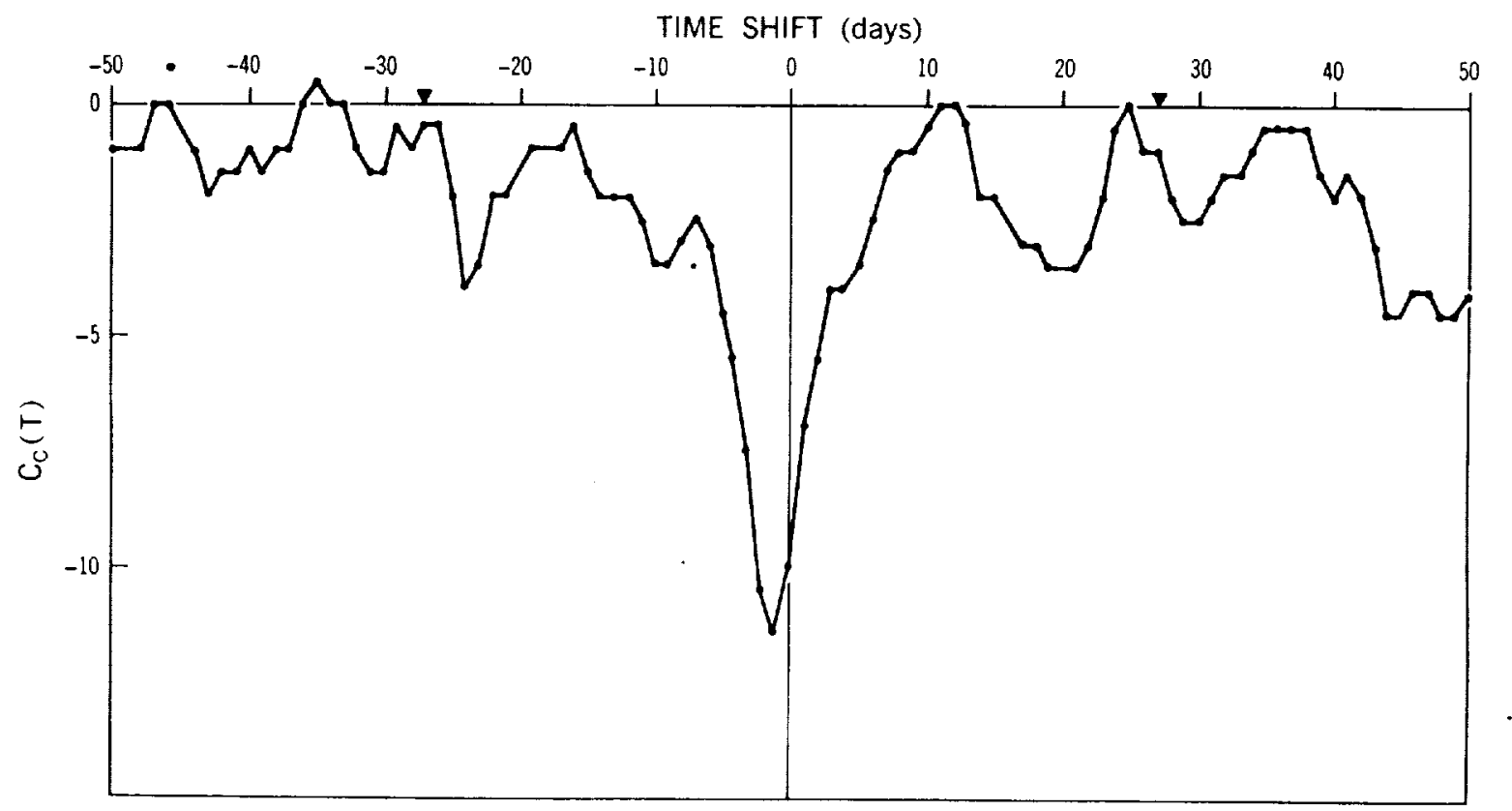

Figure 4-The cross-correlation function between magnetic activity and cosmic-ray intensity. On the right-hand side, cosmic-ray intensity precedes magnetic activity; on the left, the order is reversed. 
not conspicuous in this case. There seems to be, on the whole, quite a difference between the 27-day recurrence tendencies of cosmic radiation and that of magnetic activity (References 23 and 24). While the spectrum of cosmic-ray variations shows a single well defined peak, that of magnetic activity not only has a second harmonic of te $\mathrm{n}$ exceeding the fundamental, but also shows higher harmonics, up to the sixth (Figure 5).

\section{THE RIDIGITY DEPENDENCE OF \\ THE 27-DAY VARIATION}

Regarding the counting rate as a voltage signal, we can define the "power" contained in it, between the frequencies $f_{1}$ and $f_{2}$ as the area contained by the power-spectral density graph between the two frequencies:

$$
\text { Power }=\int_{f_{1}}^{f_{2}} P(f) d f
$$

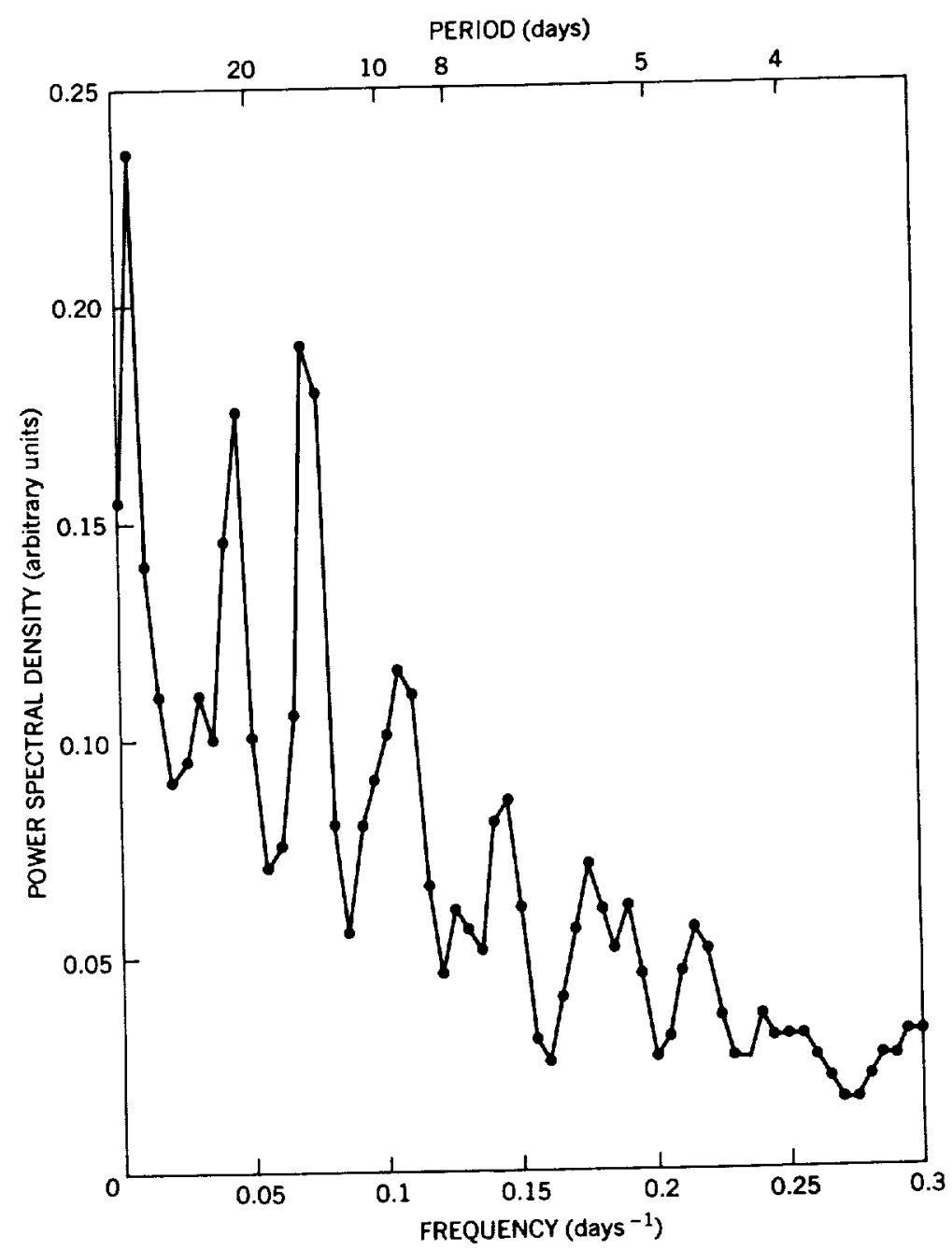

Figure 5-The power spectrum of magnetic activity indices during IGY:

The power contained in the 27-day variation will be proportional to the area enclosed by the 27-day peak. It should be noted however, that this quantity will not be obtained by passing the data through a filter which selects a limited band around 27 days. In that case, the power would also contain a major contribution from the "pedestal" upon which the peak is superimposed, which presumably is due to irregular variations. We now define as the equivalent amplitude $A$ of the variation the amplitude of a pure sinusoidal variation; containing the same power as is contained in the peak: the method by which A is calculated is described in Appendix B. The values of A thus obtained are between 0.5 and 2 percent.

The equivalent amplitudes have still to be corrected for altitude. Since the latitude dependence of the variation strongly resembles that of Forbush decreases, we adopt the correction proposed for 


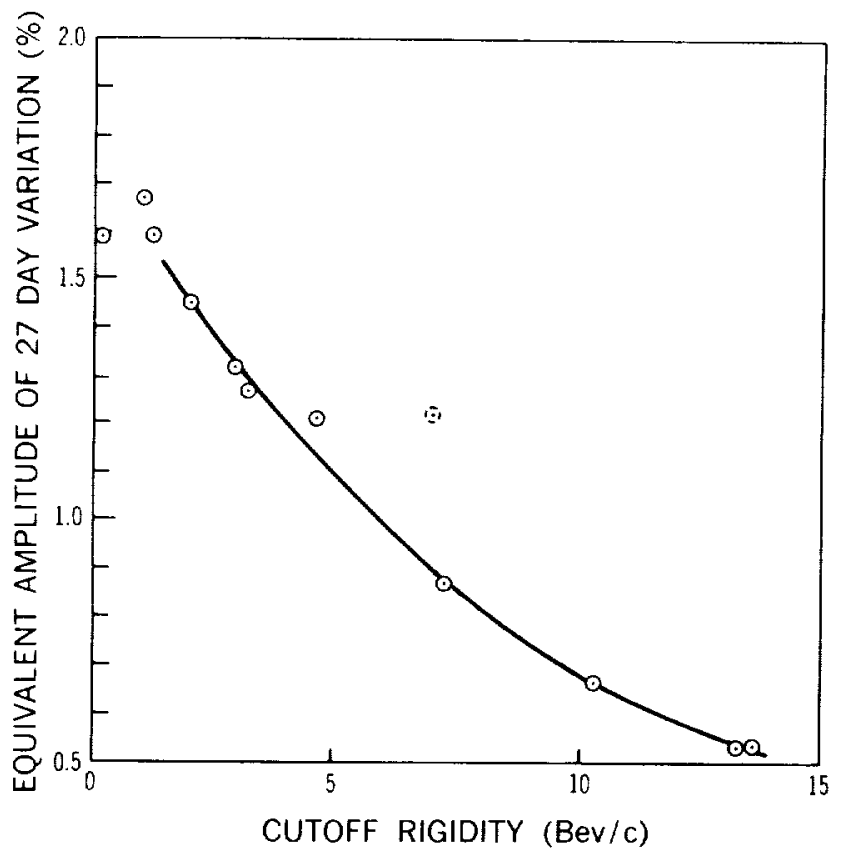

Figure 6-Latitude dependence of 27-day amplitude, averaged over IGY.

Table 1

Average Amplitudes of the 27-Day Variation for a Number of IGY Neutron Monitors.

\begin{tabular}{|l|c|c|c|}
\hline \multicolumn{1}{|c|}{ Station } & $\begin{array}{c}\text { Amplitude } \\
(\%)\end{array}$ & $\begin{array}{c}\text { Corrected } \\
\text { Amplitude } \\
(\%)\end{array}$ & $\begin{array}{c}\text { Cutoff } \\
\text { Rigidity } \\
(\mathrm{Bev} / \mathrm{c})\end{array}$ \\
\hline \hline Mawson & 1.59 & 1.59 & 0.2 \\
Sulphur Mtn. & 2.05 & 1.66 & 1.1 \\
Uppsala & 1.58 & 1.58 & 1.3 \\
Mt. Wellington & 1.62 & 1.50 & 2.0 \\
Climax & 1.84 & 1.305 & 3.0 \\
Zugspitze & 1.71 & 1.26 & 3.3 \\
Rome & 1.20 & 1.20 & 4.7 \\
Hermanus & 1.21 & 1.21 & 7.0 \\
Alma Ata & 0.94 & 0.85 & 7.3 \\
Mt. Norikura & 0.88 & 0.66 & 10.3 \\
Huancayo & 0.73 & 0.52 & 13.2 \\
Lae & 0.53 & 0.53 & 13.5 \\
\hline
\end{tabular}

the latter case by McCracken and Johns (Reference 25), amounting to 12 percent per 1000 meters. This is further justified by the fact that corrected amplitudes of some high altitude stations (e.g., Sulphur Mountain; Huancayo) fall close to those of sea level stations (e.g., Uppsala; Lae), having approximately the same cutoff. In all, 12 amplitudes were found and plotted against cutoff rigidities obtained from the eccentric dipole model by Kodama, Kondo, and Wada (Reference 26); they are given in Table 1 and in Figure 6.

It will be seen that most of the points fall on a smooth curve, with the exception of Hermanus. This station is likely to have its cutoff lowered by local anomalies in the geomagnetic field, and was therefore not considered. It will also be noted that there seems to be no flattening of the curve down to a cutoff rigidity of about $1 \mathrm{Bev} / \mathrm{c}$ - this implies that the primary radiation in the low energy region undergoes very large variations.

From the latitude dependence of the counting rate, it is possible to deduce the modulation experienced by various portions of the primary spectrum. Let

$$
\begin{aligned}
\mathbf{N}(\mathbf{p})= & \text { The sea level counting rate at cut- } \\
& \text { off rigidity } p, \\
\delta N(p)= & \text { The } 27 \text {-day equivalent amplitude, } \\
\mathbf{S}(\mathrm{p})= & \text { The primary differential proton } \\
& \text { spectrum, } \\
\delta \mathbf{S}(\mathrm{p})= & \text { The amplitude of the } 27 \text {-day varia- } \\
& \text { tion undergone by the primary } \\
& \text { spectrum at rigidity } p \text {, and }
\end{aligned}
$$

$Y(p)=$ The "gross" yield function at rigidity $p$ (Reference 27, Equation 11).

The experimental data consist of the equivalent amplitudes $A(p)=\delta N / N$, and the latitude dependence $N(p)$. Neglecting penumbral effects, we can write 


$$
\begin{gathered}
N(p)=\int_{p}^{\infty} S\left(p^{\prime}\right) Y\left(p^{\prime}\right) d p^{\prime} . \\
\delta N(p)=\int_{p}^{\infty} \delta S\left(p^{\prime}\right) Y\left(p^{\prime}\right) d p^{\prime}=N(p) A(p) .
\end{gathered}
$$

Differentiating these expressions, and dividing Equation 2 by Equation 1, we have

$$
\frac{\delta S}{S}=\frac{d}{d N}[N(p) A(p)]
$$

We now need two experimentally determined functions of the cutoff rigidity - the equivalent amplitude $A(p)$, and the total counting rate $N(p)$. Unfortunately, not many sea level measurements of the latitude dependence of $N(p)$ exist for IGY. We shall, therefore, base our calculations on the quiettime rates $N_{0}(p)$ used by Quenby and Webber (Reference 27); they are arbitrarily normalized to $\mathrm{N}_{0}(15 \mathrm{Bev})=100$.

Let us denote the ratio between the differential primary spectra during IGY and during the solar minimum (1954-5) by

$$
a(p)=\frac{S(p)}{S_{0}(p)}
$$

values of $a(p)$ are taken from the work of F. McDonald (Reference 28). The total counting rate $N(p)$ changes relatively little over the solar cycle; therefore, no correction is applied to it. On the other hand, $d N / d p$ is proportional to $\mathrm{S}(\mathrm{p})$, and receives a correction factor $a$. Substituting in Equation 3, we obtain

$$
\frac{\delta S}{S}=\mathbf{A}(\mathbf{p})+\mathbf{N}_{0}(\mathbf{p}) \frac{\frac{\mathrm{dA}}{\mathrm{dp}}}{a(p) \frac{\mathrm{dN}_{0}}{\mathrm{dp}}} .
$$

The results are presented in Table 2 and in Figure 7.

The rigidity dependence obtained here may be compared to other experimental results. It can be seen from Figure 7 that between 12.5 and $3.5 \mathrm{Bev} / \mathrm{c}$, it roughly follows a $\mathrm{p}^{-1}$ relation; this agrees with results found for Forbush events (Reference 29). The dependence

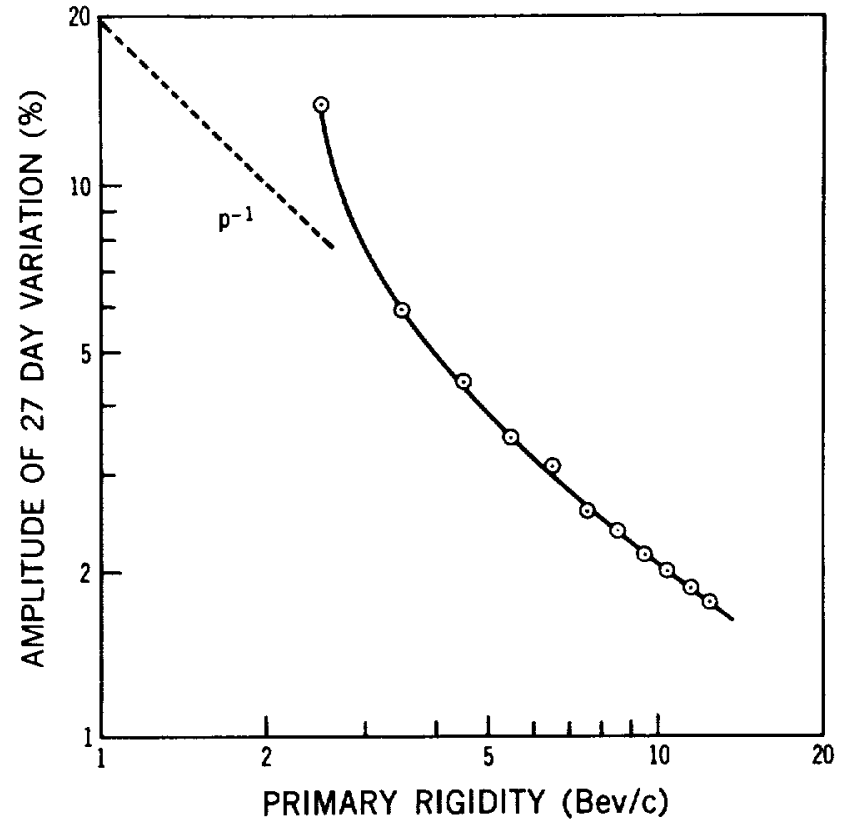

Figure 7-Rigidity dependence of the 27-day modulation undergone by the primary spectrum, averaged over IGY. 
Table 2

The Rigidity Dependence of the 27-Day Variations.

\begin{tabular}{|c|l|l|l|l|l|l|c|}
\hline $\begin{array}{c}\mathrm{p} \\
(\mathrm{Bev} / \mathrm{c})\end{array}$ & \multicolumn{1}{|c|}{$\mathrm{N}_{0}$} & $\frac{\mathrm{dN} \mathrm{N}_{0}}{\mathrm{dp}}$ & $\alpha$ & $10^{2} \mathrm{~A}(\mathrm{p})$ & $\frac{\mathrm{dA}}{\mathrm{dp}}$ & $\begin{array}{c}\delta \mathrm{S} / \mathrm{S} \\
\text { uncorrected } \\
(\%)\end{array}$ & $\begin{array}{c}\delta \mathrm{S} / \mathrm{S} \\
\text { corrected } \\
(\%)\end{array}$ \\
\hline \hline 2.5 & 172.5 & 3.2 & 0.58 & 1.38 & 0.135 & 8.79 & 14.0 \\
3.5 & 168.0 & 5.8 & 0.68 & 1.26 & 0.110 & 4.44 & 5.93 \\
4.5 & 161.3 & 7.6 & 0.76 & 1.15 & 0.115 & 3.57 & 4.38 \\
5.5 & 153.4 & 8.2 & 0.81 & 1.045 & 0.105 & 3.00 & 3.48 \\
6.5 & 145.2 & 8.1 & 0.84 & 0.935 & 0.100 & 2.73 & 3.08 \\
7.5 & 137.5 & 7.5 & 0.855 & 0.843 & 0.080 & 2.32 & 2.56 \\
8.5 & 130.3 & 6.7 & 0.87 & 0.770 & 0.070 & 2.13 & 2.34 \\
9.5 & 124.0 & 6.0 & 0.875 & 0.705 & 0.060 & 1.93 & 2.12 \\
10.5 & 118.4 & 5.2 & 0.88 & 0.648 & 0.055 & 1.90 & 1.98 \\
11.5 & 113.5 & 4.6 & 0.89 & 0.603 & 0.045 & 1.72 & 1.85 \\
12.5 & 109.1 & 4.1 & 0.895 & 0.555 & 0.040 & 1.61 & 1.74 \\
\hline
\end{tabular}

does not seem to be a power-law one, however, and it steepens at low rigidities. The rigidity dependence should also be compared with the large 27-day amplitudes found by other workers, especially by the Russians (References 30-33) at moderate balloon altitudes and high latitudes. Since balloon-borne instruments are relatively more sensitive to low rigidities, their results are in general agreement with the results obtained here. We cannot very well extrapolate the rigidity dependence to low energies; it seems, however, quite possible that the low energy cutoff of primary cosmic radiation undergoes a 27-day modulation at solar maximum, and that this may be a cause for experimental discrepancies.

\section{IRREGULAR VARIATIONS}

The 27-day peak is superimposed upon a continuum, representing the contribution of irregular variations to the power spectrum. This continuum (plotted against frequency) is roughly exponential in shape, reaching a relatively constant noise level (see Appendix B) at a frequency of about $5 /$ day.

The latitude dependence of the continuum seems to be approximately the same, at first glance, as that of the 27-day peak. This in itself is not surprising, since it is bound to contain a major contribution from Forbush events, which have practically the same latitude dependence. The comparison is complicated, however, by the fact that the continuum also contains contributions from instrumental drifts, which are not latitude dependent; this will tend to make the latitude dependence less steep than that of the 27-day variation. Because of this effect, one should discount stations in which the continuum is abnormally high.

Table 3 gives the ratio (in arbitrary units) of the power-spectral density to the area of the 27-day peak for various frequencies. If the continuum has the same latitude dependence as the 27-day 
Table 3

Latitude Dependence of Irregular Variations in Cosmic Ray Intensity During IGY.

\begin{tabular}{|c|c|c|c|c|c|c|c|}
\hline \multirow{2}{*}{ Station } & \multicolumn{7}{|c|}{$\frac{\text { Power Spectral Density }}{\text { Area of } 27 \text {-day Peak }}$} \\
\hline & $\begin{array}{l}\text { Freq }= \\
.005 \% \text { day }\end{array}$ & $.010 /$ day & $.015 /$ day & $.020 /$ day & $.055 /$ day & $.060 /$ day & $.065 /$ day \\
\hline Mawson & 1095 & 358 & 272 & 277 & 106 & 71 & 48 \\
\hline Sulfur Mtn. & 930 & 477 & 305 & 219 & 81 & 47 & 37 \\
\hline Uppsala & 734 & 409 & 341 & 292 & 91 & 70 & 55 \\
\hline Mt. Wellington & 958 & 440 & 327 & 282 & 107 & 65 & 48 \\
\hline Climax & 919 & 467 & 358 & 316 & 93 & 66 & 53 \\
\hline Zugspitze & 753 & 351 & 314 & 247 & 107 & 73 & 49 \\
\hline Rome & 989 & 425 & 281 & 232 & 109 & 75 & 52 \\
\hline Hermanus & 5635 & 1357 & 422 & 333 & 215 & 133 & 51 \\
\hline Alma Ata & 9042 & 1886 & 1108 & 810 & 316 & 181 & 89 \\
\hline Norikura & 1495 & 513 & 363 & 334 & 177 & 98 & 70 \\
\hline Huancayo & 1808 & 422 & 340 & 362 & 158 & 66 & 53 \\
\hline Lae & 2777 & 1309 & 679 & 696 & 198 & 214 & 175 \\
\hline
\end{tabular}

variation, this ratio at any given frequency should be constant for all stations. It can readily be seen that for three stations (Lae, Hermanus and Alma Ata) the ratios are unusually large; it is possible that these stations experience considerable drifts. For the other stations, there may be some increase towards the equator, but generally, the ratio seems to be fairly constant. It is, therefore, reasonable to assume that the latitude dependence of the continuum at low frequencies is close to that of the 27-day peak.

\section{CONCLUSION}

Several theories exist about the cause of the 27-day variation. One approach has been investigated in detail by Alfvèn (References 34 and 35). Alfvèn assumes that the interplanetary magnetic field near the solar equatorial plane contains "beams" of high plasma flux and magnetic field density, which co-rotate with the sun. These beams last for several rotations, and every time they intercept the earth, magnetic and cosmic-ray disturbances occur.

Alternative approaches are discussed by Dorman, who examined and rejected varlous explanations based on (1) a solar magnetic dipole noncoincident with the solar rotation axis, (2) atmospheric effects, and (3) high energy particles produced by solar flares. In Reference 37, Section 32, Paragraph $9(\mathrm{~d})$, he suggests that "the effect of the decrease in cosmic-ray intensity during the time of geomagnetic disturbances, is the basis of the phenomenon of 27-day variations of the cosmic rays." The Forbush decreases, to which Dorman refers, are obviously nonperiodic phenomena, as they can 
generally be traced back to solar flares occurring a day or two previously. However, flares tend to be associated with centers of solar activity, and these are not evenly distributed in solar longitude. As is shown by the 27-day variation of sunspot numbers, there will generally be one center much more active than the rest. Every time this center faces the earth, there is a marked tendency for sudden commencement-type magnetic storms and their associated Forbush decreases to occur, leading to an apparent 27 -day periodicity.

The results obtained here support this hypothesis. The energy dependence of the variation approximates that obtained for the Forbush effect (References 29 and 37). The variation in period length (see Figure 2) can be explained by noting that the "favorable interval" for an active area to cause a Forbush decrease is quite wide (Reference 38); the cosmic-ray record for the beginning of IGY (Figure 8), when the 27-day variation was very high, shows that the separation of the main Forbush events was indeed of the order of 27 days.

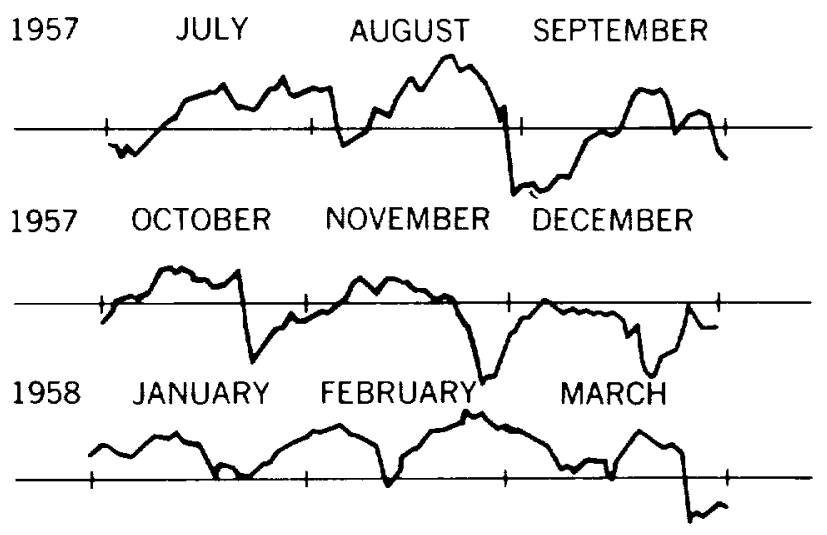

Figure 8-Cosmic-ray intensities during the first half of IGY as recorded by the Climax neutron monitor.
Examining this point more closely, we find that the main Forbush events alone cannot account for the 27-day variation. For one thing, the second harmonic of the variation would have been higher in that case. We also note that the cosmicray intensity (Figure 8 ) often starts decreasing before the main Forbush event. These "predecreases"(Reference 39) may be tentatively identified as Forbush decreases, the main impact of which misses the earth; they cause considerable smoothing of the 27-day variation, especially at high altitudes (Reference 30, Figure 1).

When we take into account the rigidity dependence of the 27-day variation obtained here,

its correlation with magnetic activity, and its time dependence, it appears very likely that this variation is associated with nonrecurrent magnetic storms, showing spurious periodicity because of their origin in solar activity centers. Perhaps recurrent magnetic storms may be responsible for a 27day variation different from the one discussed here, with presumably a smaller amplitude (possibly conforming with Alfven's model). This can only be established by analyzing periods of low solar activity when Forbush events are rare and magnetic activity shows strong recurrence tendencies (Reference 40). 


\section{REFERENCES}

1. Zwanzig, W., "Zur 27-tägigen Periode der Kosmischen Strahlung," Zeitschrift fur Naturforschung 16a(11):1237-1239, November 1961

2. Venkatesan, D., "The 27-Day Variation in Cosmic Ray Intensity," Supplemento al Nuovo Cimento $8(2): 285-297,1958$

3. Venkatesan, D., "Changes in Amplitude of the 27-day Variation in Cosmic Ray Intensity during the Solar Cycle of Activity," Tellus 10(1):117-125, February 1958

4. Schuster, A., "The Periodicity of Sun Spots," Astrophys. J. XXIII(2):101, March 1906

5. Chapman, S. and Bartels, J., "Geomagnetism," Oxford: The Clarendon Press, 1940

6. Holloway, J. L., Jr., "Smoothing and Filtering of Time Series and Space Fields," in: Advances in Geophysics, ed. by H. E. Landsberg and J. Van Mieghem, New York: Academic Press, 1958, Vol. 4, pp. 351-389

7. Chree, C., "Some Phenomena of Sunspots and of Terrestrial Magnetism at Kew Observatory," Phil. Trans. Roy. Soc. London 212A:75-116, 1913

8. Monk, A. T., and Compton, A. H., "Recurrence Phenomena in Cosmic-Ray Intensity," Rev. Mod. Phys. 11(3-4):173-179, July-October 1939

9. Broxon, J. W., "Relation of the Cosmic Radiation to Geomagnetic and Heliophysical Activities," Phys. Rev. 62(11-12):508-522, December 1 and 15, 1942

10. Lockwood, J. A., and Shea, M. A. (Univ. of New Hampshire, Physics Dept.), "The 27-Day Recurrent Variations in the Cosmic-Ray Intensity," Air Force Cambridge Research Laboratory-TN60-699, 1960

11. Kodama, M., "The 27-Day Recurrence of Cosmic-Ray Intensity at the Minimum Solar Activity," in: Proc. Moscow Cosmic Ray Conf., Moscow, 1960, Vol. 4, pp. 287-292

12. Rice, S. O., "Mathematical Analysis of Random Noise," in: Selected Papers on Noise and Stochastic Processes, ed. by N. Wax, New York: Dover, 1954, pp. 133-294

13. Welch, P. D., "A Direct Digital Method of Power Spectrum Estimation," IBM J. Res. and Dev. 5(2):141-156, April 1961

14. Bendat, J. S., "Principles and Applications of Random Noise Theory," New York: John Wiley and Sons, 1958

15. Davenport, W. B., and Root, W. L., "An Introduction to the Theory of Random Signals and Noise," New York: McGraw-Hill, 1958

16. Hannan, E. J., "Time Series Analysis," London: Methuen, 1960 
17. Solodovnikov, V. V., "Introduction to the Statistical Dynamics of Automatic Control Systems," Transl. ed. by J. B. Thomas and L. A. Zadeh, New York: Dover, 1960

18. Blackman, R. B., and Tukey, J. W., "The Measurement of Power Spectra," New York: Dover, $1 \varepsilon=i w$

19. Van Isacker, J., "Generalized Harmonic Analysis," in Advances in Geophys., ed. by H. E. Landsberg and J. Van Mieghem, New York: Academic Press, 1961, Vol. 7, pp. 189-214

20. Wiener, N., "Extrapolation, Interpolation and Smoothing of Stationary Time Series," New York: John Wiley and Sons, 1949

21. Page, C. H., "Instantaneous Power Spectra," J. Appl. Phys. 23(1):103-106, January 1952

22. Fonger, W. H., "Cosmic Radiation Intensity-Time Variations and Their Origin. II: Energy Dependence of 27-Day Variations," Phys. Rev. 91(2):351-361, July 15, 1953

23. Panofsky, H., Lethbridge, M. D., and Neuberger, H., "Research Directed Towards the Study of the Relation of Solar Energy Variations to Changes of the Tropospheric Circulations," Penn. State Univ., Mineral Industries Exper. Sta., Final Rept. on Contract AF 19(604)-2251 (AFCRC-TR-58263) June 30, 1958

24. Ward, F.W., Jr., "The Variance (Power) Spectra of Ci, Kp, and Ap," J. Geophys. Res. 65(8):23592373, August 1960

25. McCracken, K. G., and Johns, D. H., "The Attenuation Length of the High Energy Nucleonic Component of the Cosmic Radiation near Sea Level," Nuovo Cimento 13(1):96-107, July 1, 1959

26. Kodama, M., Kondo, I., and Wada, M., "Cut-off Rigidities of Cosmic-Ray Particles Calculated for the Eccentric Dipole Model of the Earth's Magnetic Field," J. Scientific Research Institute (Tokyo) 51(1455-1464):138-157, September 1957

27. Webber, W. R., and Quenby, J. J., "On the Derivation of Cosmic Ray Specific Yield Functions," Phil. Mag. 4(41):654-664, May 1959

28. McDonald, F. B., "Primary Cosmic-Ray Intensity near Solar Maximum," Phys, Rev. 116(2):462463 , October 15, 1959

29. Lockwood, J. A., "An Investigation of the Forbush Decreases in the Cosmic Radiation," J. Geophys. Res. 65(12):3859-3880, December 1960

30. Vernov, S. N., Tulinov, V. F., and Charakhch'ian, "The 27-Day Cosmic-Ray Period in the Stratosphere," Doklady Akademii Nauk SSSR 122(5):788-791, October 11, 1958; Translation in Soviet Phys.-Doklady 3(5):980-982, September-October 1958

31. Pomerantz, M. A., Agarwal, S. P., and Potnis, V. R., "Direct Observation of Periodic Variation of Primary Cosmic-Ray Intensity," Phys. Rev. 109(1):224-225, January 1, 1958 
32. Pomerantz, M. A., Agarwal, S. P., and Potnis, V. R., "Balloon Flight Investigations of Primary Cosmic Rays During Solar Disturbances," in: Proc. Moscow Cosmic Ray Conf., Moscow, 1960, Vol. 4, pp. 65-73

33. Vernov, S. N., Samosudov, B. E., et al., "Studies of Intensity Variations of Cosmic Radiation in the Stratosphere," in: Proc. Moscow Cosmic Ray Conf., Moscow, 1960, Vol. 4, pp. 53-64

34. Alfvén, H., "The Sun's General Magnetic Field," Tellus 8(1):1-12, February 1956

35. Alfvén, H., "Interplanetary Magnetic Field," in: Electromagnetic Phenomena in Cosmical Physics, ed. by B. Lehnert, Cambridge: The University Press, 1958, pp. 284-292

36. McCracken, K. G., "Energy Dependence of Transient Changes in the Primary Cosmic-Ray Spectrum," Phys. Rev. 117(6):1570-1579, March 15, 1960

37. Dorman, L. I., "Cosmic Ray Variations," Moscow: State Publishing House for Technical and Theoretical Literature, 1957 (Translation by Technical Documents Liaison Office, WrightPatterson Air Force Base, 1958)

38. Sinno, K., "Mechanism of Cosmic Ray Storms Inferred from Some Statistical Results," in: Proc. Internat. Conf. on Cosmic Rays and the Earth Storm, Kyoto, September 1961. II. Joint Sessions, Tokyo: Physical Society of Japan, 1962, pp. 395-399

39. Legrand, J. P., "The Cosmic Rays 'Predecreases' (Prebaisse) in the Maximum Solar Activity Period (April 1957-December 1958)" in: Proc. Moscow Cosmic Ray Conf., Moscow, 1960, Vol. 4, pp. 219-227

40. Tandon, J. N., "Cosmic Ray Increases during the Sunspot Minimum of 1953," J. Atmos. Terrest. Phys. 21(2/3):203-205, June 1961

41. Japan, Science Council, National Committee for the International Geophysical Year, "CosmicRay Intensity during the International Geophysical Year," Nos. 1-3, 1959-1960 

Appendix A

\section{Estimation of the Fluctuation Noise}

In practice, the cosmic-ray neutron rate $x(t)$ is estimated at fixed intervals $\Delta t$. Since the number of counts in any interval is subject to statistical fluctuation, this estimation is subject to error. We now wish to find the extent to which this will affect the estimation of power-spectral density $P(f)$.

Instead of dealing with an imperfectly sampled signal $x(t)$, it is found convenient to analyze a signal $x^{\prime}(t)=x(t)+n(t)$ which is sampled without error-where $n(t)$ is the noise, duplicating the effects of statistical fluctuations. Since the measurements consist of averaging the counting rate over time intervals $\Delta t$, we choose $n(t)$ to be a "histogram" function (see Figure B1), each column of which has the width $\Delta t$. We assume that the heights of the columns are normally distributed around the mean; actually, they obey a Poissonian distribution, but it is only at very high sampling rates, when the average number of counts per interval is small, that the distinction is significant. Henceforth, we shall assume that both $x(t)$ and $n(t)$ have been normalized to average zero.

The noise is correlated with itself only for points within the same column; hence, its autocorrelation function is:

$$
C_{n}(t)= \begin{cases}\overline{n_{0}^{2}}(1-t / \Delta t) & \text { for } t<\Delta t \\ 0 & \text { for } t>\Delta t .\end{cases}
$$

The noise-power spectrum is then

$$
\begin{aligned}
P_{n}(f) & =\int_{-\infty}^{\infty} e^{-2 \pi i f t} C_{n}(t) d t \\
& =\overline{2 n^{2}} \int_{0}^{\Delta t} \cos 2 \pi f t\left(1-\frac{t}{\Delta t}\right) d t \\
& =\overline{n^{2}} \Delta t[\text { dif }(f \Delta t)]^{2}
\end{aligned}
$$

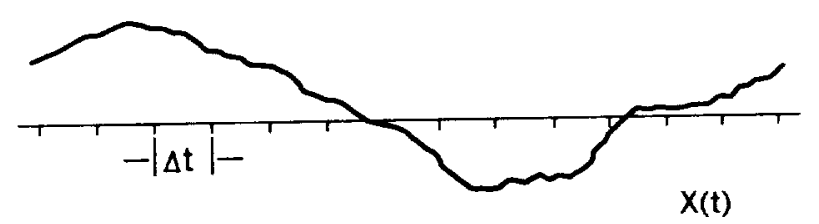

where

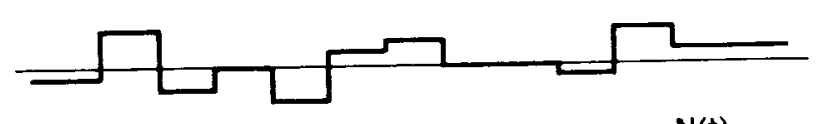

$$
\text { dif } x=\frac{\sin \pi x}{\pi x}
$$

is the well-known diffraction function. 
Since the signal and noise are uncorrelated, the autocorrelation of $x^{\prime}(t)$ will be the sum of those of the signal and of the noise, and the power spectrum will retain this additive property. The noise spectrum $P_{n}(f)$ is thus superimposed upon the desired spectrum. This in itself would cause no trouble if we had an infinite run of data at hand, since the true spectrum can then be obtained by subtracting the calculated value of $P_{n}(f)$. In a finite run, however, $P_{n}(f)$ undergoes unpredictable fluctuations, as derived from a $x^{2}$ distribution (Reference 18 ), and it is this variability which constitutes the noisiness introduced by statistical fluctuations.

For the low frequency end of the spectrum, we may approximate dif $(\mathrm{f} \Delta t) \approx 1$. Let us assume that the cosmic-ray rate shows no variation apart from statistical fluctuations. Then, defining $w, N_{0}$, and $\mathrm{N}$, as in Appendix A, we have

$$
\overline{n^{2}}=(\overline{W-\bar{W}})^{2}
$$

and

$$
\overline{N_{1}^{2}}=\left(\frac{N_{0}}{1000}\right)^{2} \overline{\left(n^{2}\right)}
$$

For purely statistical fluctuations, however,

$$
\overline{N_{1}^{2}}=N_{0},
$$

hence,

$$
\overline{\mathrm{n}^{2}}=\frac{10^{6}}{\mathrm{~N}_{0}}
$$

The noise level will then be

$$
P_{n}(f) \approx \frac{10^{6} \Delta t}{N_{0}}
$$

The result is evidently inversely proportional to the counting rate. In actual practice, it seems to be somewhat larger than the above estimate indicates, probably because of nonstatistical fluctuations.

For daily averages of a typical neutron monitor station, such as Rome, $\mathrm{N}_{0}=7.5 \times 10^{5}$. If time is measured in days, the noise level turns out to be of the order of unity. This is completely negligible in comparison to the amplitude of the 27-day peak, which on the same scale is close to 4500 . 
Appendix B

\section{Power Spectra of Logarithmically Reduced Data}

The IGY data used in most of the present computation (Reference 41) do not give the counting rate $\mathrm{N}$, but instead give

$$
W(t)=1000 \log \left(\frac{N}{N^{*}}\right)=1000 \log N+\text { constant },
$$

where $\mathrm{N}^{*}$ is a conveniently chosen constant. If we regard the counting rate as the sum of a constant rate $\mathrm{N}_{0}$ and a small fluctuation $\mathrm{N}_{1}$, averaging zero, we get (by expanding)

$$
\begin{aligned}
W & \approx 1000 \frac{N_{1}}{N_{0}}+\text { constant } \\
\overline{N_{1}^{2}} & =\left(\frac{N_{0}}{1000}\right)^{2}(\overline{W-\bar{W}})^{2}, \\
& =\left(\frac{N_{0}}{1000}\right)^{2} \mathrm{C}(0), \\
& =\left(\frac{N_{0}}{1000}\right)^{2} \int_{-\infty}^{\infty} P(f) d f, \\
& =2\left(\frac{N_{0}}{1000}\right)^{2} \int_{0}^{\infty} P(f) d f
\end{aligned}
$$

where $C(t)$ and $P(f)$ are the autocorrelation and the spectral density obtained by analyzing $W(t)$. The power contained in any finite frequency band of $N(t)$ is proportional to the integral of $P$ over the band, with the same proportionality factor as above.

Assume a frequency peak of area $Q$; if it were entirely due to a harmonic variation of frequency $f$ and amplitude $a$, its power would equal $a^{2 / 2}$. Even if the peak is not infinitely sharp, we can define an equivalent amplitude a containing the same power; then

$$
\left(\frac{\mathrm{a}}{\mathrm{N}_{0}}\right)^{2}=4 \times 10^{-6} \mathrm{Q} \text {. }
$$

In the text, relative equivalent amplitudes $A=a / N_{0}$ are usually given. 
